\title{
Heart Failure During Hospitalization
}

National Cancer Institute

\section{Source}

National Cancer Institute. Heart Failure During Hospitalization. NCI Thesaurus. Code C80477.

Clinical diagnosis of heart failure during a hospitalization, of which heart failure was not the admitting diagnosis. 